\title{
Résultats, après 10 ans, d'une coupe à diamètre minimum d'exploitation dans un peuplement feuillu
}

\author{
par
}

\author{
Lise Robitaille ${ }^{1}$ et Jean-Louis Boivin ${ }^{1}$
}

\section{Résumé}

Une coupe à diamètre minimum d'exploitation, où l'on devait récolter seulement les bouleaux jaunes de $43 \mathrm{~cm}$ et plus et les autres essences de $27 \mathrm{~cm}$ et plus de d.h.p., s'est soldée par un prélèvement de 56 p. 100 du volume marchand pour l'ensemble du peuplement. Dix ans après ce prélèvement, une excellente régénération s'était établie; elle était constituée de 53 p. 100 de bouleau jaune, soit une proportion de près du double de celle trouvée dans le peuplement original; elle commençait à mal se développer sous couvert. L'acroissement en volume marchand de ce couvert a été faible au cours des dix ans qui ont suivi la coupe; il a presque tout été produit par des arbres de qualité impropre au sciage et au déroulage; il était à l'image du peuplement avant et après coupe, qui était de piètre qualité; tout indique que cette qualité va encore diminuer.

\begin{abstract}
A diameter-limit cut, where limits were $43 \mathrm{~cm}$ for yellow birch and $27 \mathrm{~cm}$ for other species, resulted in the harvesting of $56 \%$ of the merchantable volume of the whole stand. Ten years after this treatment, excellent regeneration was established; yellow birch accounted for $53 \%$ of this regeneration, which is nearly double that found in untreated stands; it had begun to have a slower development under forest cover. The merchantable volume increment of the residual stand has been slow during the ten years after the cut, being almost entirely produced by low-grade trees so that the residual stand looks like the original one where quality will continue to decrease.
\end{abstract}

\section{Introduction}

Une coupe à diamètre minimum d'exploitation, qui a débuté en 1972 dans un vieux peuplement de faible qualité à dominance d'érable à sucre, bouleau jaune et hêtre à grandes feuilles dans la forêt de Duchesnay, s'est poursuivie en 1973 et 1974. Cette coupe s'est traduite dans les faits par une coupe d'intensité variable. Pour mieux en étudier les effets sur la régénération et l'accroissement des arbres, en plus de présenter les résultats globaux pour les secteurs traités et témoins, nous les donnons par groupes d'intensité du volume résiduel, soit moins de 30 p. 100,30 à 70 p. 100 et plus de 70 p. 100.

Cette étude s'est faite à l'aide d'un suivi de places d'étude permanentes établies et mesurées avant coupe, et remesurées immédiatement après coupe, puis cinq et dix ans plus tard, aussi bien dans la partie du peuplement coupée que dans la partie soustraite à la coupe et conservée comme témoin. Notre rapport compare les résultats obtenus dans les deux parties du peuplement et obtenus avec les différentes intensités de coupe.

\section{Aire d'étude et méthodes}

L'expérience s'est déroulée sur le territoire de la Station forestière de Duchesnay qui a pour coordonnées : $46^{\circ} 53^{\prime}$ de 1Service de la recherche appliquée, Ministère de l'Energie et des Ressources,
2700, rue Einstein, Sainte-Foy (Québec) G1P 3W8 latitude nord et $71^{\circ} 40^{\prime}$ de longitude ouest. Cette station forestière couvre une superficie de 8910 hectares et est située à environ 40 kilomètres au nord-ouest de la ville de Québec.

Le secteur étudié couvrant une superficie de 366 ha, se trouve à la limite sud de la section forestière Laurentienne (L.4a de Rowe, 1972) dans le domaine de l'érablière à bouleau jaune. La carte des régions écologiques du Québec méridional (Thibault, 1985) précise que ce secteur fait partie du sous-domaine de l'érablière à bouleau jaune et hêtre $(3 \mathrm{~g})$.

Le sol est constitué de podzols reposant sur des tills et le drainage varie de rapide à moyen. La topographie est assez accidentée.

Le peuplement original était une vieille érablière à bouleau jaune et hêtre dont l'âge des tiges variait entre 1 et 250 ans. Au début de l'expérience, la partie coupée était en tout semblable à la partie témoin. On trouvait avant coupe, un volume marchand de $223 \mathrm{~m}^{3} /$ ha constitué de $34 \mathrm{p} .100$ d'érable à sucre (Acer saccharum Marsh.), de 25 p. 100 de bouleau jaune (Betula alleghaniensis Britt.), de 37 p. 100 de hêtre à grandes feuilles (Fagus grandifolia Ehrh.) et d'un peu d'érable rouge (Acer rubrum L.), d'épinette rouge (Picea rubens Sarg.), de pruche de l'Est (Tsuga canadensis [L.] Carr.) et de sapin baumier (Abies balsamea [L.] Mill.). Le volume était réparti sur 361 tiges marchandes d'un d.h.p. moyen de 29,8 cm; cependant, 78 p. 100 de ces tiges étaient de qualité impropre au bois d'oeuvre. 
En 1972, 1973 et 1974, on a établi au dispositif expérimental comprenant 78 places d'étude circulaires traitées et témoins. Sur l'ensemble de la superficie de chaque parcelle de 0,08 ha, on a mesuré le d.h.p. et la classe de qualité de tous les arbres de grosseur marchande, c'est-àdire de ceux qui avaient $9 \mathrm{~cm}$ et plus de d.h.p. Chaque arbre de $19 \mathrm{~cm}$ et plus de d.h.p. était classé A, B, C ou D selon le pourcentage de bois d'oeuvre qu'on pouvait en retirer, l'arbre de qualité $D$ ne pouvant donner aucun bois d'oeuvre (Lavallée, 1968; Lavallée et Lortie, 1968).

Sur la moitié de la superficie de chaque parcelle, les gaules de 1 à $9 \mathrm{~cm}$ de d.h.p. étaient mesurées et pointées par classe de diamètre. Quant aux semis de $30 \mathrm{~cm}$ de hauteur à $1 \mathrm{~cm}$ de d.h.p. et de 1 à $30 \mathrm{~cm}$ de hauteur, leur présence était notée, par essence, dans cinq quadrats circulaires d'une superficie de $4 \mathrm{~m}^{2}$ dans chaque place d'étude.

Lors de la compilation des données, on a regroupé les places d'étude traitées en trois groupes, en tenant compte du pourcentage du volume résiduel de bois sur pied après coupe. Les places sévèrement coupées, où il restait moins de $30 \mathrm{p}$. 100 du volume initial, c'est-à-dire $45 \mathrm{~m}^{3} / \mathrm{ha}$ en moyenne de bois marchand, couvraient environ 25 p. 100 de la superficie coupée; les places fortement coupées, où le volume résiduel variait de 30 à 70 p. 100 , soit en moyenne $101 \mathrm{~m}^{3} / \mathrm{ha}$, couvraient 60 p. 100 de la superficie traitée et les places faiblement coupées, dont le volume résiduel était supérieur à 70 p. 100 , soit $195 \mathrm{~m}^{3} / \mathrm{ha}$, couvraient $15 \mathrm{p} .100$ de la superficie traitée.

On a effectué toutes les compilations et présenté les résultats en tenant compte de ces trois intensités de coupe, sévère, forte et faible, et en les comparant à l'ensemble des secteurs traités et témoins dont les volumes résiduels étaient respectivement de 100 et de $223 \mathrm{~m}^{3}$ ha.

Les coefficients de distribution des semis et le nombre de gaules par hectare ont été calculés. On a finalement fait de même pour le volume marchand toutes qualités puis par classe de qualité, pour le nombre d'arbres et pour le diamètre moyen. Tous ces calculs ont été faits pour les données de chacun des quatre mesurages effectués. En comparant les résultats obtenus avant coupe, immédiatement après coupe, puis 5 à 10 ans plus tard, on a pu constater les changements survenus.

\section{Résultats}

\section{La régénération}

Nous ne présentons les résultats que pour la classe de dimensions de régénération la plus représentative à chaque moment de l'étude. Pour l'année de la coupe, nous avons retenu celle de 1 à $30 \mathrm{~cm}$ de hauteur (tableau 1), puis celle de $30 \mathrm{~cm}$ de hauteur à $1 \mathrm{~cm}$ de d.h.p. 5 ans après coupe (tableau 2) et celle de 1 à $9 \mathrm{~cm}$ de d.h.p. 10 ans après coupe (tableau 3).

Immédiatement après coupe, la régénération était sensiblement partout la même quelle que soit l'intensité du traitement (tableau 1); les principales essences d'ombre, l'érable à sucre et le hêtre, étaient alors présentes dans près de 50 p. 100 des quadrats sur l'ensemble du dispositif expérimental.

Cinq ans après coupe, on observait une régénération mieux distribuée et plus variée excepté dans les places témoins où le volume résiduel de bois sur pied était plus élevé (tableau 2). On notait donc des différences importantes selon les volumes résiduels. Le nombre d'essences avait augmenté dans les places coupées par rapport aux places témoins. Cette augmentation était surtout remarquable chez les essences semi-tolérantes à l'ombre telles que le bouleau jaune, l'érable rouge, le bouleau à papier (Betula papyrifera Marsh.), l'érable à épis (Acer spicatum Lam.) et l'érable de Pennsylvanie (Acer pensylvanicum L.). Quelques essences de lumière, dont le peuplier à grandes dents (Populus grandidentata Michx.), le peuplier faux-tremble (Populus tremuloides Michx.) et le cerisier de Pennsylvanie (Prunus pensylvanica I.f.), avaient fait leur apparition dans les places où la coupe avait été sévère.

Dix ans après coupe, la distribution et la variation de la régénération avaient les mêmes proportions que cinq ans après coupe sauf pour le bouleau jaune qui prédominait alors dans le secteur coupé (tableau 3 ). Les gaules de bouleau jaune prédominaient par leurs dimensions, leur nombre et leur proportion par rapport aux autres essences, qui augmentaient au même rythme que l'intensité de la coupe. Le bouleau jaune était suivi par les essences arbustives dont les dimensions, le nombre et la proportion par rapport aux autres essences variaient relativement peu selon l'intensité de coupe; dans les places témoins, les essences arbustives étaient peu abondantes.

\section{L'accroissement}

Si le volume résiduel variait beaucoup entre les différents traitements immédiatement après coupe, tel n'était pas le cas de la proportion des essences qui le constituaient (tableau 4).

Les abres impropres au bois d'oeuvre formaient 64 p. 100 de ce volume de tous les traitements regroupés (tableau 5); leur proportion augmentait de 57 à 76 p. 100 de la coupe sévère à la coupe faible. Un peu plus de la moitié provenait d'arbres qui étaient au-dessus du diamètre minimum et qui n'avaient pas été coupés; le volume de ces arbres était à 85 p. 100 impropre au bois d'oeuvre. II faut dire qu'avant coupe, 87 p. 100 du volume des arbres au-dessus du diamètre minimum était impropre au bois d'oeuvre. Ce sont 248 arbres d'un diamètre moyen de $25,2 \mathrm{~cm}$ qui composaient les $100 \mathrm{~m}^{3}$ / ha de l'aire coupée à diamètre minimum; ce sont 361 arbres de $29,8 \mathrm{~cm}$ qui donnaient les $223 \mathrm{~m}^{3}$ ha de l'aire témoin.

Cinq ans après coupe, les volumes présents variaient encore beaucoup selon les volumes laissés sur pied lors de la coupe (tableau 6). L'accroissement dans l'aire coupée n'a été que de $2 \mathrm{~m}^{3} /$ ha comparativement à $3 \mathrm{~m}^{3} /$ ha dans l'aire témoin. N'eut été de la mortalité très forte survenue dans les places faiblement coupées où l'on avait laissé sur pied un volume de $195 \mathrm{~m}^{3} / \mathrm{ha}$, la coupe à diamètre minimum aurait produit un meilleur accroissement que le témoin; cette mortalité est survenue à peu près également chez toutes les essences. La composition, la proportion d'arbres impropres au bois d'oeuvre, le nombre d'arbres et le diamètre moyen n'avaient pas changé non plus au cours des premiers cinq ans qui ont suivi la coupe.

Dix ans après coupe, l'écart entre les volumes variait encore beaucoup car le volume de l'ensemble de la partie du peuplement coupé à diamètre minimum ne s'était accrue que 100 à $115 \mathrm{~m}^{3} /$ ha alors que celui de la partie témoin s'était accru de 223 à $232 \mathrm{~m}^{3} /$ ha (tableau 7 ). Ceci représente un gain de seulement $6 \mathrm{~m}^{3} / \mathrm{ha}$ dû à la coupe en 10 ans. Ce gain a 
Tableau 1. Coefficient de distribution * des semis de 1 à $30 \mathrm{~cm}$ de hauteur par traitement pour les principales essences, immédiatement après coupe.

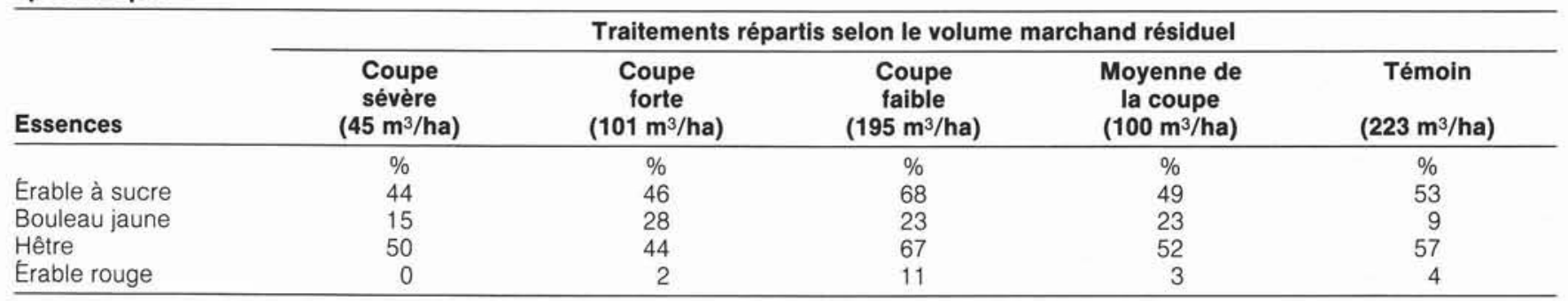

- Le coefficient de distribution se définit comme étant le pourcentage de quadrats oủ au moins une tige de l'essence étudiée est présente.

Tableau 2. Coefficient de distribution des semis de $30 \mathrm{~cm}$ de hauteur à $1 \mathrm{~cm}$ de d.h.p. par traitement pour toutes les essences, cinq ans après coupe.

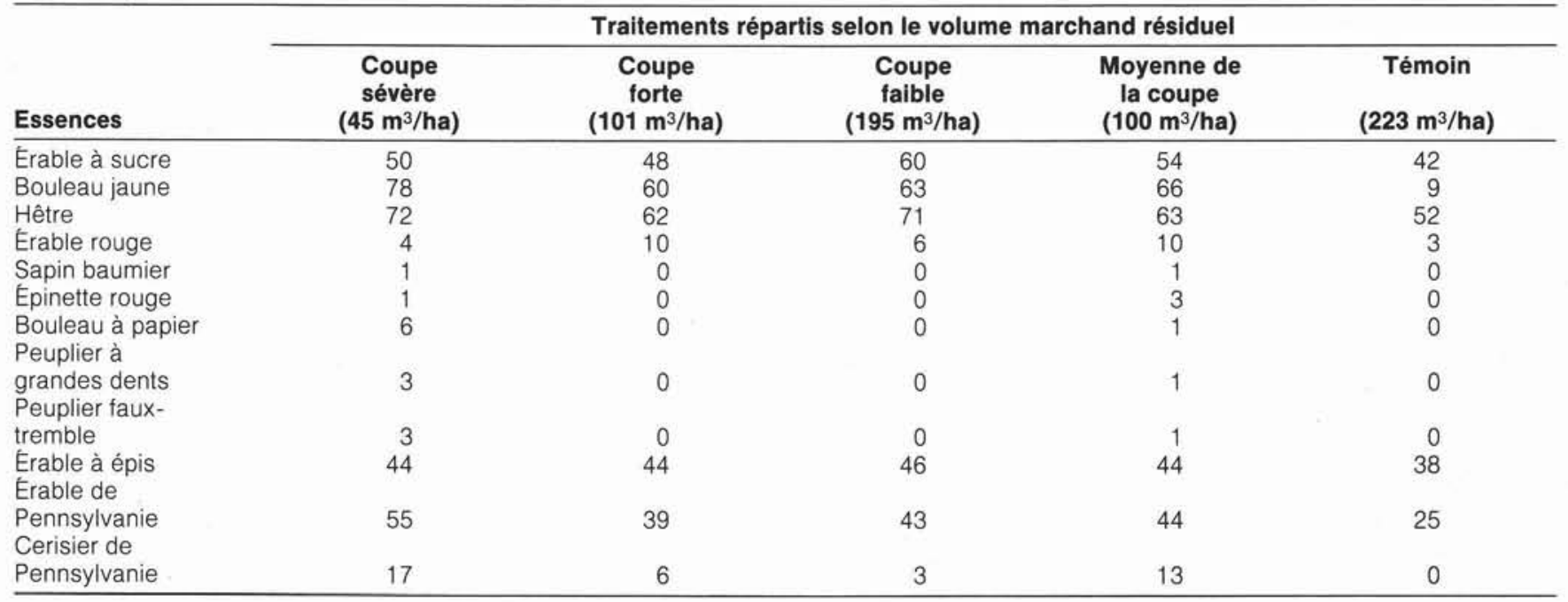

Tableau 3. Nombre de gaules de 1 à $9 \mathrm{~cm}$ de d.h.p. par hectare par traitement pour les principales essences dix ans après coupe.

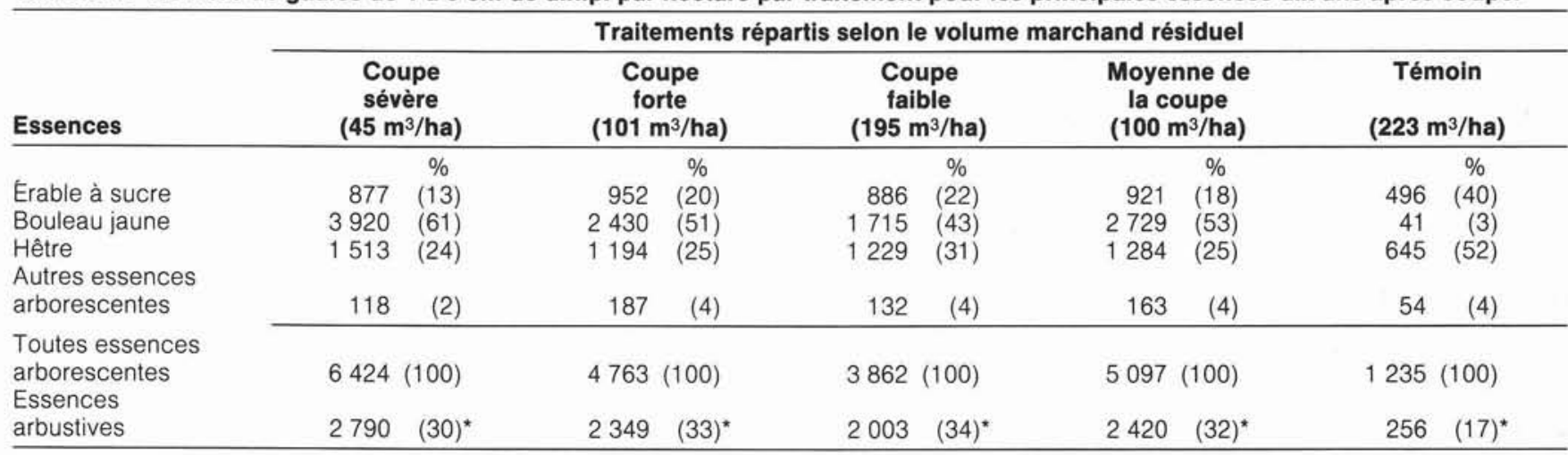

-Pourcentage de toutes les essences arborescentes et arbustives.

Tableau 4. Volume marchand brut, en $\mathrm{m}^{3} / \mathrm{ha}$ et en $\%$, par traitement pour les principales essences, immédiatement après coupe.

\begin{tabular}{|c|c|c|c|c|c|c|c|c|c|c|}
\hline \multirow{3}{*}{$\begin{array}{l}\text { Essences } \\
\text { Erable à sucre } \\
\text { Bouleau jaune } \\
\text { Hêtre }\end{array}$} & \multicolumn{10}{|c|}{ Traitements répartis selon le volume marchand résiduel } \\
\hline & \multicolumn{2}{|c|}{$\begin{array}{l}\text { Coupe } \\
\text { sévère } \\
\left(45 \mathrm{~m}^{3} / \mathrm{ha}\right)\end{array}$} & \multicolumn{2}{|c|}{$\begin{array}{c}\text { Coupe } \\
\text { forte } \\
\left(101 \mathrm{~m}^{3} / \mathrm{ha}\right)\end{array}$} & \multicolumn{2}{|c|}{$\begin{array}{c}\text { Coupe } \\
\text { faible } \\
\left(195 \mathrm{~m}^{3} / \mathrm{ha}\right)\end{array}$} & \multicolumn{2}{|c|}{$\begin{array}{l}\text { Moyenne de } \\
\text { la coupe } \\
\left(100 \mathrm{~m}^{3} / \mathrm{ha}\right)\end{array}$} & \multicolumn{2}{|c|}{$\begin{array}{c}\text { Témoin } \\
\text { (223 } \mathrm{m}^{3 / h a)}\end{array}$} \\
\hline & $\begin{array}{l}\mathrm{m}^{3} / \mathrm{ha} \\
17 \\
10 \\
16 \\
\end{array}$ & $\begin{array}{r}\% \\
(38) \\
(23) \\
(36) \\
\end{array}$ & $\begin{array}{l}\mathrm{m}^{3} / \text { ha } \\
24 \\
35 \\
37\end{array}$ & $\begin{array}{c}\% \\
(24) \\
(35) \\
(37) \\
\end{array}$ & $\begin{array}{l}\mathrm{m}^{3} / \mathrm{ha} \\
69 \\
48 \\
71\end{array}$ & $\begin{array}{c}\% \\
(35) \\
(25) \\
(32)\end{array}$ & $\begin{array}{l}\mathrm{m}^{3} / \mathrm{ha} \\
29 \\
30 \\
36\end{array}$ & $\begin{array}{c}\% \\
(29) \\
(30) \\
(36)\end{array}$ & $\begin{array}{l}\mathrm{m}^{3} / \mathrm{ha} \\
75 \\
55 \\
82\end{array}$ & $\begin{array}{c}\% \\
(34) \\
(25) \\
(37)\end{array}$ \\
\hline Toutes essences & 45 & $(100)$ & 101 & $(100)$ & 195 & $(100)$ & 100 & $(100)$ & 223 & $(100)$ \\
\hline
\end{tabular}


Tableau 5. Répartion en pourcentage du volume par essence et par classe de qualité du volume total de chaque essence.

Tiges au-dessus du diamètre minimum d'exploitation

\begin{tabular}{|c|c|c|c|c|c|c|c|c|}
\hline \multirow{2}{*}{ Essences } & \multicolumn{4}{|c|}{$\begin{array}{c}\text { Avant coupe } \\
\text { Classes de qualité }\end{array}$} & \multicolumn{4}{|c|}{$\begin{array}{l}\text { Immédiatement après coupe } \\
\text { Classes de qualité }\end{array}$} \\
\hline & A & B & C & D & A & B & C & D \\
\hline Erable à sucre & 2,4 & 2,7 & 6,9 & 87,9 & 7,0 & 6,9 & 11,6 & 74,5 \\
\hline Bouleau jaune & 2,3 & 6.4 & 9,5 & 81,8 & - & 11,7 & 6,8 & 81,5 \\
\hline Hêtre à grandes feuilles & 0,7 & 3,2 & 6,6 & 89,5 & 1,0 & 2,2 & 5,3 & 91,5 \\
\hline Toutes & 2,3 & 3,6 & 6,9 & 87,2 & 3,1 & 4,8 & 7,4 & 84,7 \\
\hline
\end{tabular}

Tiges au-dessous du diamètre minimum d'exploitation

\begin{tabular}{lrrrrrrrr} 
& & \multicolumn{3}{c}{ Avant coupe } & & \multicolumn{3}{c}{ Immédiatement après coupe } \\
\cline { 2 - 9 } & A & B & C & D & A & B & C & D \\
\hline Erable à sucre & 17,5 & 22,5 & 19,4 & 38,2 & 14,4 & 30,5 & 26,5 & 16,7 \\
Bouleau jaune & 17,8 & 20,0 & 24,6 & 37,6 & 19,6 & 19,4 & 22,3 & 18,9 \\
Hêtre à grandes feuilles & 5,4 & 23,3 & 13,1 & 58,1 & 4,9 & 25,5 & 18,0 & 8,0 \\
$\quad$ Toutes & 15,9 & 21,7 & 21,6 & 40,7 & 15,8 & 23,6 & 22,8 & 14,0
\end{tabular}

Toutes les tiges

\begin{tabular}{lrrrrrrrr} 
& & \multicolumn{2}{c}{ Avant coupe } & \multicolumn{3}{c}{ Immédiatement après coupe } \\
& A & B & C & D & A & B & C & D \\
\hline Erable à sucre & 4,2 & 5,0 & 8,3 & 82,4 & 9,5 & 14,9 & 16,7 & 58,9 \\
Bouleau jaune & 9,3 & 12,6 & 16,3 & 61,7 & 15,3 & 17,7 & 18,9 & 48,1 \\
Hètre à grandes feuilles & 1,2 & 5,3 & 7,2 & 86,3 & 1,9 & 7,2 & 8,0 & 82,9 \\
$\quad$ Toutes & 5,0 & 7,2 & 9,9 & 77,8 & 8,6 & 12,9 & 14,0 & 64,5 \\
\hline
\end{tabular}

tLortie et Lavallèe

Tableau 6. Volume marchand brut, en $\mathrm{m}^{3} / \mathrm{ha}$ et en $\%$, par traitement pour les principales essences, cinq ans après coupe.

\begin{tabular}{|c|c|c|c|c|c|c|c|c|c|c|}
\hline \multirow{3}{*}{$\begin{array}{l}\text { Essences } \\
\text { Érable à sucre }\end{array}$} & \multicolumn{10}{|c|}{ Traitements répartis selon le volume marchand résiduel } \\
\hline & \multicolumn{2}{|c|}{$\begin{array}{c}\text { Coupe } \\
\text { sévère } \\
\left(45 \mathrm{~m}^{3} / \mathrm{ha}\right)\end{array}$} & \multicolumn{2}{|c|}{$\begin{array}{c}\text { Coupe } \\
\text { forte } \\
\left(101 \mathrm{~m}^{3} / \mathrm{ha}\right)\end{array}$} & \multicolumn{2}{|c|}{$\begin{array}{c}\text { Coupe } \\
\text { faible } \\
\left(195 \mathrm{~m}^{3} / \mathrm{ha}\right)\end{array}$} & \multicolumn{2}{|c|}{$\begin{array}{c}\text { Moyenne de } \\
\text { la coupe } \\
\left(100 \mathrm{~m}^{3} / \mathrm{ha}\right)\end{array}$} & \multicolumn{2}{|c|}{$\begin{array}{c}\text { Témoin } \\
\text { (223 } \mathrm{m}^{3 / \mathrm{ha}} \text { ) }\end{array}$} \\
\hline & $\begin{array}{l}\mathrm{m}^{3} / \mathrm{ha} \\
23 \\
14 \\
15\end{array}$ & $\begin{array}{r}\% \\
(44) \\
(27) \\
(29)\end{array}$ & $\begin{array}{l}\mathrm{m}^{3} / \mathrm{ha} \\
28 \\
39 \\
37\end{array}$ & $\begin{array}{r}\% \\
(26) \\
(36) \\
(34)\end{array}$ & $\begin{array}{l}\mathrm{m}^{3} / \mathrm{ha} \\
69 \\
48 \\
71\end{array}$ & $\begin{array}{r}\% \\
(35) \\
(25) \\
(32)\end{array}$ & $\begin{array}{l}\mathrm{m}^{3} / \mathrm{ha} \\
31 \\
32 \\
35\end{array}$ & $\begin{array}{r}\% \\
(30) \\
(31) \\
(34)\end{array}$ & $\begin{array}{l}\mathrm{m}^{3} / \mathrm{ha} \\
74 \\
57 \\
86\end{array}$ & $\begin{array}{r}\% \\
(34) \\
(25) \\
(38)\end{array}$ \\
\hline Toutes essences & 52 & $(100)$ & 108 & $(100)$ & 188 & $(100)$ & 100 & $(100)$ & 223 & $(100)$ \\
\hline
\end{tabular}

Tableau 7. Volume marchand brut, en $\mathrm{m}^{3} / \mathrm{ha}$ et en $\%$, par traitement pour les principales essences, dix ans après coupe.

\begin{tabular}{|c|c|c|c|c|c|c|c|c|c|c|}
\hline \multirow{3}{*}{$\begin{array}{l}\text { Essences } \\
\text { Érable à sucre } \\
\text { Bouleau jaune } \\
\text { Hêtre }\end{array}$} & \multicolumn{10}{|c|}{ Traitements répartis selon le volume marchand résiduel } \\
\hline & \multicolumn{2}{|c|}{$\begin{array}{l}\text { Coupe } \\
\text { sévère } \\
\left(45 \mathrm{~m}^{3} / \mathrm{ha}\right)\end{array}$} & \multicolumn{2}{|c|}{$\begin{array}{c}\text { Coupe } \\
\text { forte } \\
\left(101 \mathrm{~m}^{3} / \mathrm{ha}\right)\end{array}$} & \multicolumn{2}{|c|}{$\begin{array}{c}\text { Coupe } \\
\text { faible } \\
\left(195 \mathrm{~m}^{3} / \mathrm{ha}\right)\end{array}$} & \multicolumn{2}{|c|}{$\begin{array}{l}\text { Moyenne de } \\
\text { la coupe } \\
\left(100 \mathrm{~m}^{3} / \mathrm{ha}\right)\end{array}$} & \multicolumn{2}{|c|}{$\begin{array}{c}\text { Témoin } \\
\text { (223 m/ha) }\end{array}$} \\
\hline & $\begin{array}{l}\mathrm{m}^{3} / \mathrm{ha} \\
29 \\
19 \\
16\end{array}$ & $\begin{array}{r}\% \\
(44) \\
(29) \\
(26)\end{array}$ & $\begin{array}{l}\mathrm{m}^{3} / \mathrm{ha} \\
35 \\
42 \\
38\end{array}$ & $\begin{array}{r}\% \\
(29) \\
(35) \\
(32)\end{array}$ & $\begin{array}{l}\mathrm{m}^{3} / \mathrm{ha} \\
71 \\
44 \\
64\end{array}$ & $\begin{array}{r}\% \\
(38) \\
(24) \\
(35)\end{array}$ & $\begin{array}{l}\mathrm{m}^{3} / \mathrm{ha} \\
39 \\
36 \\
36\end{array}$ & $\begin{array}{r}\% \\
(34) \\
(31) \\
(31)\end{array}$ & $\begin{array}{l}\mathrm{m}^{3} / \mathrm{ha} \\
78 \\
57 \\
86\end{array}$ & $\begin{array}{r}\% \\
(34) \\
(25) \\
(37)\end{array}$ \\
\hline Toutes essences & 66 & $(100)$ & 120 & $(100)$ & 185 & $(100)$ & 115 & $(100)$ & 232 & $(100)$ \\
\hline
\end{tabular}

été surtout dû à l'érable à sucre dont la représentation a légèrement augmenté dans la partie du peuplement coupé, pour atteindre celle du peuplement témoin. La mortalité a été égale dans les deux parties du peuplement avec $15 \mathrm{~m}^{3} / \mathrm{ha}$, soit d'une quantité égale à l'accroissement de la partie coupée. De ces $15 \mathrm{~m}^{3} /$ ha d'accroissement, 13 provenaient d'arbres impropres au bois d'oeuvre. Le nombre d'arbres par hectare dans la partie coupée avait augmenté de 198, dont 179 étaient de l'érable à sucre alors que le diamètre moyen avait fléchi de 25,2 à 19,5 cm; dans la partie non coupée, on notait une baisse de 35 arbres et une hausse de $1,7 \mathrm{~cm}$ du diamètre moyen au cours des 10 ans d'étude.

\section{Discussion}

L'ouverture du couvert forestier consécutive à la coupe à diamètre minimum n'a pas cessé de se faire sentir sur la régénération au cours de cette période de dix années d'étude. Ce sont le bouleau jaune, l'érable à épis et l'érable de Pennsylvanie, trois essences de tolérance intermédiaire à l'ombre, qui ont montré la réaction positive la plus forte. Mais alors que la réaction positive du bouleau jaune culminait là où il ne restait plus ou presque plus de couvert, celle des deux autres essences changeait peu selon l'intensité d'ouverture de la voûte foliacée. Ces résultats indiquent que la coupe à 
blanc de petites superficies, genre groupes, trouées ou bandes (Boivin, 1985), donnerait une meilleure régénération du bouleau jaune que la coupe progressive d'une intensité de 50 p. 100; ils indiquent de plus qu'une telle coupe progressive serait préférable à une coupe prélevant seulement 10 à 20 p. 100 du volume des arbres mûrs et surannés dans les peuplements semblables de feuillus nordiques à Duchesnay. La plupart des autres études, impliquant diverses intensités et méthodes de coupe dans les feuillus d'ombre du Québec et d'ailleurs, corrobrent ces résultats (Linteau 1948; Eyre et Zillgitt 1953; Leak et Wilson 1958; Marquis 1964; Hatcher 1966; Marquis 1967; Tubbs et Metzger 1969; Leak et Filip 1977; Roberge 1977; Robitaille 1978; Metzger 1980; Kelty et Nyland 1981; Boivin 1985).

\section{Conclusion}

Le peuplement coupé à diamètre minimum était de très mauvaise qualité et composé d'arbres d'âge, de hauteur et de diamètres variés et de relativement peu de bouleau. Avant coupe, ce peuplement avait $185 \mathrm{~m}^{3} /$ ha de bois au-dessus du diamètre minimum, dont 87 p. 100 en arbres impropres au bois d'oeuvre et $38 \mathrm{~m}^{3} /$ ha de bois au-dessous au diamètre minimum, dont 41 p. 100 en arbres impropres au bois d'oeuvre. Après coupe, il restait $65 \mathrm{~m}^{3} / \mathrm{h}$ a de bois au-dessus du diamètre minimum, dont 85 p. 100 en arbres impropres au bois d'oeuvre et $35 \mathrm{~m}^{3} /$ ha de bois au-dessous du diamètre minimum dont 38 p. 100 en arbres impropres au bois d'oeuvre; la proportion de bouleau jaune avait augmenté de 5 p. 100. La coupe n'avait donc que très faiblement amélioré la qualité et la composition du peuplement.

L'accroissement en volume consécutif à la coupe a été très faible et 87 p. 100 de cet accroissement s'est fait sur des arbres impropres au bois d'oeuvre. La proportion de bouleau jaune ne s'est accrue que de 1 p. 100. La trop forte intensité de coupe, sa très grande variabilité, l'état irrégulier du peuplement et l'arrivée massive de recrues d'érable à sucre ne laissent entrevoir aucun espoir d'amélioration ni de la qualité, ni de la composition de ce peuplement. L'excellente régénération du bouleau jaune présente est irrémédiablement condamnée à mourir d'oppression sous ce couvert d'arbres de très mauvaise qualité qui va se refermer graduellement. On a tout lieu de croire que ce qui est survenu et va survenir dans le peuplement à la suite de cette coupe ne sera qu'une répétition en pire des coupes passées semblables. Tourney et Korstran (1947), Westveld (1949), Baker (1950), Troup (1952), Hawley at Smith (1954), Biesterfeldt (1981) et Vézina et Roberge (1981) s'accordent pour affirmer que des coupes comme celles effectuées dans le peuplement sous étude conduisent à sa dégradation.

La coupe à diamètre limite, bien qu'elle favorise la régénération du bouleau jaune par les trouées pratiquées ne donne pas de résultats uniformes. Cette coupe est soit trop sévère, là où tous les arbres sont au-dessus du diamètre limite, soit trop légère là où les arbres sont petits; d'ailleurs, rien n'est prévu pour dégager les jeunes arbres ou éviter les trop grandes trouées. De plus, l'exploitant ne respecte pas les normes car il coupe surtout les meilleures tiges et laisse sur pied des gros arbres de mauvaise qualité. La faible densité du couvert résiduel favorise le chablis. II s'agit en fait d'une coupe d'écrémage.

\section{Remerciements}

Nos remerciements vont au Dr Marcien Roberge du Service canadien de Forêts pour avoir revu le texte et nous avoir fait des suggestions très appréciées.

\section{Références}

Baker, F.S. 1950. Principles of Silviculture. McGraw Hill, New York.

Biesterfeldt, R.C. (ed.) 1981. Choices in silviculture for American forests. American Society of Foresters, Washington, D.C.

Boivin, J.-L. 1985. Coupes par bandes dans des peuplements de feuillus. Résultats après 14 ans. For. Chron. 61: 229-232.

Eyre, F.H. et W.M. Zillgitt, 1953. Partial cuttings in northern hardwoods of the Lake States: Twenty years experimental results. USDA. Forest Serv., Lake States For. Exp. Sta., Tech. Bull. 1076.

Hatcher, R.J. 1966. Yellow birch regeneration on scarified seedbeds under small canopy openings. For. Chron. 42: 350-358.

Hawley, R.C. et D.M. Smith. 1954. The Practice of Silviculture, Sixth Edition. John Wiley and Sons, New York.

Kelty, M.J. et R.D. Nyland. 1981. Regenerating Adirondak northern hardwoods by shelterwood cutting and control of deer density. J. For. 79: 22-26.

Lavalée, A. 1968. Détermination de la qualité de l'érable à sucre d'après des signes apparents de carie. For. Chron. 44(4): 5-10.

Lavalée, A. et M. Lortie. 1968. Relationships between external features and trunk rot in living yellow birch. For. Chron. 44(2): 513.

Leak, W.B. et R.W. Wilson. 1958. Regeneration after cutting of old growth northern hardwoods in New Hampshire. USDA For. Serv., Northeast. For. Exp. Sta., Sta. Paper 103.

Leak, W.B. et S.M. Filip. 1977. Thirty eight years of group selection in New England northern hardwoods. J. For. 75: 641-643.

Linteau, A. 1948. Factors affecting germination and early survival of yellow birch in Québec. For. Chron. 24: 27-86.

Marquis, D.A. 1964. Regeneration of birch and associated hardwoods three years after patch cutting. USDA For. Serv., Northeast. For. Exp. Sta., Res. Paper NE-32.

Marquis, D.A. 1967. Clearcutting in northern hardwoods: Results after 30 years. USDA For. Serv., Northeast. For. Exp. Station, Res. Pap. NE 85.

Metzger, F.T. 1980. Strip clearcutting to regenerate northern hardwoods. USDA For. Serv., Northeast. For. Exp. Sta., Res. Pap. NC 186.

Roberge, M.R. 1977. Influence of cutting methods on natural and artificial regeneration of yellow birch in Québec northern hardwoods. Can. J. For. Res. 7: 175-182.

Robitaille, L. 1978. Résultats quinquennaux de plantation et de régénération naturelle du bouleau jaune suite à une coupe rase avec et sans scarification. Symposium de I'IUFRO, Nancy, 1978

Rowe, J.S. 1972. Régions forestières du Canada. Ser. Can. Forêts, Publ. $1300 \mathrm{~F}$.

Thibault, M. 1985. Carte des régions écologiques du Québec méridional. Service de la recherche et Service de la cartographie. Ministère de l'Énergie et des Ressources.

Toumey, J.W. et C.F. Korstian. 1947. Foundation of Silviculture upon an Ecological Basis. John Wiley and Sons, New York.

Troup, R.S. 1952. Silvicultural System. Second Edition. Oxford Univ. Press, London.

Tubbs, C.H. et F.T. Metzger. 1969. Regeneration of northern hardwoods under shelterwood cutting. For. Chron. 45: 333337.

Vézina, P.E. et M.E. Roberge. 1981. Comment aménager nos forêts. Les Presses de I'Université Laval, Québec.

Westveld, R.H. 1949. Applied Silviculture in the United States, Second Edition. John Wiley and Sons, New York. 